\title{
Ergänzung und Berichtigung zu
}

\section{„Zurückführung des Großwetters auf solare Erscheinungen“".}

(Diese Zeitschrift Serie A, Band I, S. 358, 1949.)

Von

Franz Baur, Bad Homburg v. d. H.

In Tabelle 2 der in der Überschrift genannten vorläufigen Mitteilung, auf S. 363, sind folgende Änderungen vorzunehmen: Die Zahl der Monate von Januar 1761 bis Februar 1947 beträgt 2234, nicht 2134. Davon entfallen 608 , nicht 508, auf die Zeit von 0,8 bis 6,0 Jahre nach einem Sonnenflecken-Maximum. Dadurch wird der Erwartungswert von sehr warmen Monaten in Mitteleuropa in diesen Abschnitt des Sonnenfleckenzyklus 27,8 (statt bisher 23,2). Infolge dieser Änderung des Erwartungswertes in der letzten Spalte wird $\chi^{2}=27,8$ und $\chi^{2}: m=3,97$. Die obere Zufallsgrenze wird also, bei richtiger Rechnung, noch viel mehr übersehritten, als es in der ersten Darstellung schien.

Tabelle 1 auf S. 363 weist in fünf Klassen einen Erwartungswert auf, der - wenn auch nicht viel - unter 5 liegt. Dadurch sind, wie in dem Aufsatz bereits erwähnt, die Voraussetzungen zur Anwendung des $\chi^{2}$ Kriteriums genau genommen nicht erfüllt. Der Beweis, daß die in der Tabelle dargestellte Verteilung der sehr kalten Wintermonate in Boston und New Haven über den Sonnenfleckenzyklus nicht mehr als zufällige Besonderheit des benützten Zeitabschnitts bei an sich bestehender Unabhängigkeit gedeutet werden kann, wird daher besser mit Hilfe des R. A. FIsHerschen H-Verfahrens [1] geführt.

Dieses Verfahren ist eine der besonders von der englischen statistischen Schule gepflegten Echtheitsprüfungen (Tests of significance). Die Fragestellung, die es beantwortet, ist folgende: In einer Reihe von $n_{1}$ Beobachtungen ist ein bestimmtes Ereignis óder Merkmal $E x_{1}$-mal, in einer zweiten Reihe von $n_{2}$-Beobachtungen $x_{2}$-mal festgestellt worden. Wie groß ist die (totale) Wahrscheinlichkeit $H$, dạß den beiden Reihen die gleiche Ereigniswahrscheinlichkeit $p$ zugrunde liegt? Das FisHersche 
Verfahren gestattet, diese Gesamtwahrscheinlichkeit $H$ zu berechnen, und gibt damit die Grundlage für das Kriterium: Wenn $H\left(x_{1}, x_{2} ; n_{1}\right.$, $\left.n_{2}\right)<\varepsilon$, so kann man annehmen, daß die beobachteten relativen Häufigkeiten $\frac{x_{1}}{n_{1}}$ und $\frac{x_{2}}{n_{2}}$ auf verschiedenen Grundwahrscheinlickeiten $p_{1}$ und $p_{2}$ beruhen. Die englische Schule wählt vielfach als Zufallsgrenze der Restwahrscheinlichkeit $\varepsilon=0,01$ oder sogar 0,05 . Wir halten es mit S. KoLLER [2] für richtiger, grundsätzlich an der strengeren Forderung $\varepsilon=0,0027$ festzuhalten [3]. Den Nachweis der Widerspruchsfreiheit und der Unabhängigkeit des FrsHerschen Verfahrens von der Größe der hypothetischen Grundwahrscheinlichkeit $p$ hat M. P. GEPPERT erbracht [4].

Wir wiederholen hier den Kern der Tabelle 1 der in Rede stehenden Arbeit und bezeichnen die Klassen mit den römischen Ziffern I bis IX:

\begin{tabular}{|c|c|c|c|c|c|c|c|c|}
\hline I & II & III & $\mathrm{IV}$ & $\mathrm{V}$ & $\mathrm{VI}$ & VII & VIII & IX \\
\hline $\begin{array}{c}2,9 \mathrm{~J} . \\
\mathrm{n} . \mathrm{Max} \text {. bis } \\
2,5 \mathrm{~J} . \\
\text { vor Min. }\end{array}$ & $\begin{array}{c}2,4 \text { bis } 1,6 \\
\text { Jahre } \\
\text { vor Min. }\end{array}$ & $\begin{array}{c}1,5 \text { bis } 0,7 \\
\text { Jahre } \\
\text { vor Min. }\end{array}$ & $\begin{array}{c}0,6 \text { vor } \\
\text { bis } \\
1,0 \text { Jahre } \\
\text { nach Min. }\end{array}$ & $\begin{array}{c}1,1 \mathrm{~J} \text {. } \\
\text { n. Min. bis } \\
1,6 \mathrm{~J} \text {. } \\
\text { vor Max }\end{array}$ & $\begin{array}{c}1,5 \text { bis } 0,8 \\
\text { Jahre } \\
\text { vor Max. }\end{array}$ & $\begin{array}{c}0,7 \text { vor } \\
\text { bis } \\
0,2 \text { Jahre } \\
\text { nach Max. }\end{array}$ & $\begin{array}{c}0,3 \text { bis } 1,2 \\
\text { Jahre } \\
\text { nach Max. }\end{array}$ & $\begin{array}{c}1,3 \text { bis } 2,8 \\
\text { Jahre } \\
\text { nach Max. }\end{array}$ \\
\hline $\begin{array}{l}2^{*} \\
8,13\end{array}$ & $\begin{array}{l}9 \\
4,38\end{array}$ & $\begin{array}{l}0^{*} \\
4,24\end{array}$ & $\begin{array}{l}17 \\
8,27\end{array}$ & $\begin{array}{l}4^{*} \\
9,26\end{array}$ & $\begin{array}{l}7 \\
4,31\end{array}$ & $\begin{array}{l}1^{*} \\
4,88\end{array}$ & $\begin{array}{l}9 \\
4,88\end{array}$ & $\begin{array}{l}7 \\
7,64\end{array}$ \\
\hline $\begin{array}{c}115 \\
0,145\end{array}$ & $\begin{array}{c}62 \\
0,078\end{array}$ & $\begin{array}{c}60 \\
0,076\end{array}$ & $\begin{array}{c}117 \\
0,148\end{array}$ & $\begin{array}{c}131 \\
0,165\end{array}$ & $\begin{array}{c}61 \\
0,077\end{array}$ & $\begin{array}{c}69 \\
0,087\end{array}$ & $\begin{array}{c}69 \\
0,087\end{array}$ & $\begin{array}{c}108 \\
0,135\end{array}$ \\
\hline
\end{tabular}

Die Anwendung des Fishazschen $H$-Verfahrens auf je zwei Klassen obiger Tabelle, wobei die in der ersten Zeile gegebenen absoluten Häufigkeiten der sehr kalten Monate die $x_{1}$ und $x_{2}$, die in der dritten Zeile gegebenen absoluten Häufigkeiten aller Monate die $n_{1}$ und $n_{2}$ liefern, ergibt:

\begin{tabular}{|c|c|c|c|c|c|c|c|}
\hline \multicolumn{3}{|c|}{ Klassen } & $x_{i}$ & $x_{2}$ & $n_{1}$ & $n_{2}$ & $H\left(x_{1}, x_{2} ; n_{1}, n_{2}\right)$ \\
\hline $\mathbf{I}$ & und. & II & 2 & 9 & 115 & 62 & 0,0015 \\
\hline $\mathrm{I}$ & , & IV & 2 & 17 & 115 & 117 & 0,00025 \\
\hline I & , & VIII & 2 & 9 & 115 & 69 & 0,00278 \\
\hline II & , & III & 9 & 0 & 62 & 60 & 0,0017 \\
\hline II & , & VII & 9 & 1 & 62 & 69 & 0,0074 \\
\hline III & , & IV & 0 & 17 & 60 & 117 & 0,00057 \\
\hline III & , & VIII & 0 & 9 & 60 & 69 & 0,00276 \\
\hline IV & " & $\mathrm{V}$ & 17 & 4 & 117 & 131 & 0,0011 \\
\hline IV & , & VII & 17 & 1 & 117 & 69 & 0,0019 \\
\hline
\end{tabular}

Wie man sieht, ergeben sich bei sechs Paaren von Klassen für das Bestehen einer gleich großen Wahrscheinlichkeit des Eintretens sehr kalter Wintermonate Wahrscheinlichkeiten, die kleiner als 0,0027 sind. Damit ist erwiesen, daß das Eintreten sehr kalter Wintermonate in 
344 F. BAdR: Zurückführung des Großwetters auf solare Erscheinungen.

Boston und New Haven von der Lage des Monats im Sonnenfleckenzyklus nicht unabhängig ist. Das Endergebnis ist das gleiche wie mit dem $\chi^{2}$-Kriterium, aber die Grundlage ist exakter.

\section{Literaturverzeichnis.}

1. Fisher, R. A.: Statistical methods for research workers. 5. Aufl. London 1934, S. 99-101; 7. Aufl. London 1938, S. 101-102.

2. Koller, S.: Graphische Tafeln zur Beurteilung statistischer Zahlen. 2. Aufl. Dresden und Leipzig 1943.

3. BAUR, F.: Einführung in die Großwetterkunde. Wiesbaden 1948, S. 140 bis 141 .

4. GePPeRr, M. P.: Über den Vergleich zweier beobachteter Häufigkeiten. Habilitationsschrift. Verlag S. Hirzel, Leipzig 1942. 